\title{
Performance Evaluation of Wireless Sensor Networks for Different Radio Models Considering Mobile Event
}

\author{
Tao Yang $\dagger$, Makoto Ikeda $\dagger$, Leonard Barolli $\ddagger$, Arjan Durresi $\dagger \dagger$, Fatos Xhafa $\dagger \ddagger$ \\ $\dagger$ Graduate School of Engineering \\ Fukuoka Institute of Technology (FIT) \\ 3-30-1 Wajiro-Higashi, Higashi-Ku, Fukuoka 811-0295, Japan \\ E-mail: $\{$ bd07003, bd07001\}@bene.fit.ac.jp \\ $\ddagger$ Department of Information and Communication Engineering \\ Fukuoka Institute of Technology (FIT) \\ 3-30-1 Wajiro-Higashi, Higashi-Ku, Fukuoka 811-0295, Japan \\ E-mail: barolli@ fit.ac.jp \\ $\dagger †$ Department of Computer and Information Science \\ Indiana University Purdue University at Indianapolis (IUPUI) \\ 723 W. Michigan Street SL 280 Indianapolis, IN 46202, USA \\ E-mail: durresi@cs.iupui.edu \\ $\dagger \ddagger$ Department of Languages and Informatics Systems \\ Technical University of Catalonia \\ C/Jordi Girona 1-3, 08034 Barcelona, Spain \\ E-mail: fatos@1si.upc.edu
}

\begin{abstract}
In this paper, we consider the behavior of a wireless sensor network for TwoRayGround and Shadowing radio models for the case of mobile event. By means of simulations, we analyse the performance of AODV protocol. In the previous work, we considered that the event node is stationary in the observation field. In this work, we want to investigate how the sensor network performs in the case when the event node moves. The simulation results show that the shadowing phenomena, by destroying the regularity of the network, reduce the mean distance among nodes and at the same time increase the interference level and the latency of packet transmission. We found that for mobile event, the Goodput and routing efficiency of TwoRayGround is better than Shadowing, but the Depletion of Shadowing is better than TwoRayGround.
\end{abstract}

\section{Introduction}

In recent years, technological advances have lead to the emergence of distributed Wireless Sensor Networks (WSNs) which are capable of observing the physical world, processing the data, making decisions based on the observations and performing appropriate actions. These networks can be an integral part of systems such as battle-field surveillance and microclimate control in buildings, nuclear, biological and chemical attack detection, home automation and environmental monitoring $[1,2]$.

Wireless sensor network simulation is an important part of the current research. A large number of algorithms were first implemented and evaluated using several network simulators like ns-2. Most MANET routing protocols have been developed and tested in that fashion, and only later they evolved towards real world implementations.

Recently, there are many research works for sensor networks [3, 4]. In our previous work [5], we implemented a simulation system for sensor networks consider different 
protocols ( e.g: AODV, DSR, DSDV, OLSR. ) and different propagation radio models. In [6], we analysised the performance of the WSNs considering different topologies with the irregular radio model. Also, we analysised the performance of our proposed simulation systems. But, we considered that the event node is stationary in the observation field. However, in many applications the event node may move. For example, in an ecology environment the animals may move randomly. Another example is when an event happens in a robot or in a car.

In this work, we want to investigate how the sensor network performs in the case when the event moves and use also Shadowing propagation model. We carry out simulations for lattice topology, TwoRayGround and Shadowing radio model considering Ad-hoc On-demand Distance Vector (AODV) protocol. We compare the simulation results for the mobile event using different propagation radio model. The simulation results have shown that the goodput for the case of mobile event node is better than the stationary event node using AODV protocol and TwoRayGround model. Also, the goodput for the mobile event node case does not change too much compared with the stationary event case using AODV and Shadowing model, but the goodput is not good when the number of nodes is increased.

The paper is organized as follows. In Sections 2, we explain the proposed network simulation model, simulation topology, routing protocols and radio models. In Secton 3, we introduce the event detection and transport. In Section 4, we introduce the goodput, Routing Efficiency (RE) and energy depletion concepts. In Section 5 we present simulation results. Finally, conclusions of the paper are given in Section 6.

\section{Proposed Network Simulation Model}

Proposed network simulation model is shown in Fig. 1. In our WSNs model, every node detects the physical phenomenon and sends back to the sink node data packets. We suppose that the sink node is more powerful than sensor nodes and it is always located at the borders of the service area. This model can be used for remote monitoring of hazard or inaccessible areas [7]. We analyse the performance of the network in a fixed time interval. This can be considered as the available time for the detection of the phenomenon and its value is application dependent. In this paper, we consider that a mobile event is moving randomly in the WSNs field. In Fig. 2 is shown one pattern of movement event's path. We implemented a simulation system for WSNs considering moving event using ns- 2 . We evaluated the goodput, routing efficiency and consumed energy of AODV protocol for TwoRayGround and Shadowing propagation models in case of the lattice topology.

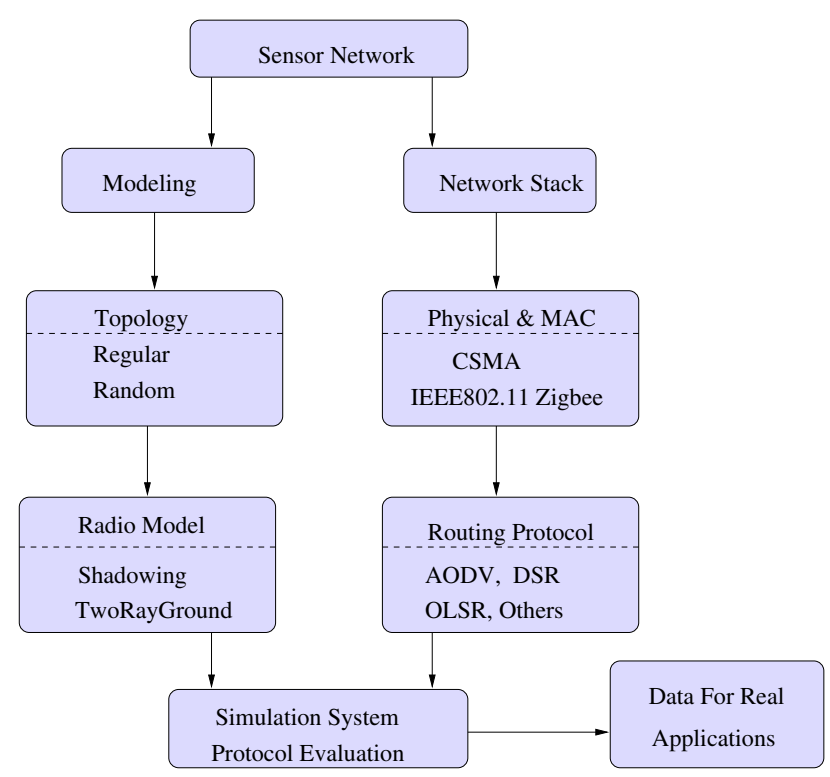

\section{Figure 1. Proposed network simulation model.}

\subsection{Topology}

For the physical layout of the WSNs, two types of deployment has been studied so far: the random and the lattice deployment. In the former, nodes are supposed to be randomly distributed, while in the latter nodes are vertexes of particular geometric shape, e.g. a square grid. For space constraints, we present results for the square grid topology only. In this case, in order to guarantee the connectedness of the network we should set the transmission range of every node to the step size, $d$, which is the minimum distance between two rows (or columns) of the grid. In fact, by this way the number of links that every node can establish, a.k.a the node degree is $D=4$. By using Cooper's theorem [8] along with some power control techniques, one could use also $D=2^{1}$. However, we assume all nodes to be equal and then the degree is fixed to 4 . Nodes at the borders have $D=2$.

\subsection{Routing Protocol}

We are aware of many proposals of routing protocols for ad-hoc networks during recent years. Here, we consider AODV protocol [9]. The AODV is an improvement of DSDV to on-demand scheme. It minimize the broadcast packet by creating route only when needed. Every node in network should maintain route information table and participate in routing table exchange. When source node wants

\footnotetext{
${ }^{1}$ By using the theorem in [8], we can say that a simple 2 regular network is almost surely strongly 2 connected.
} 


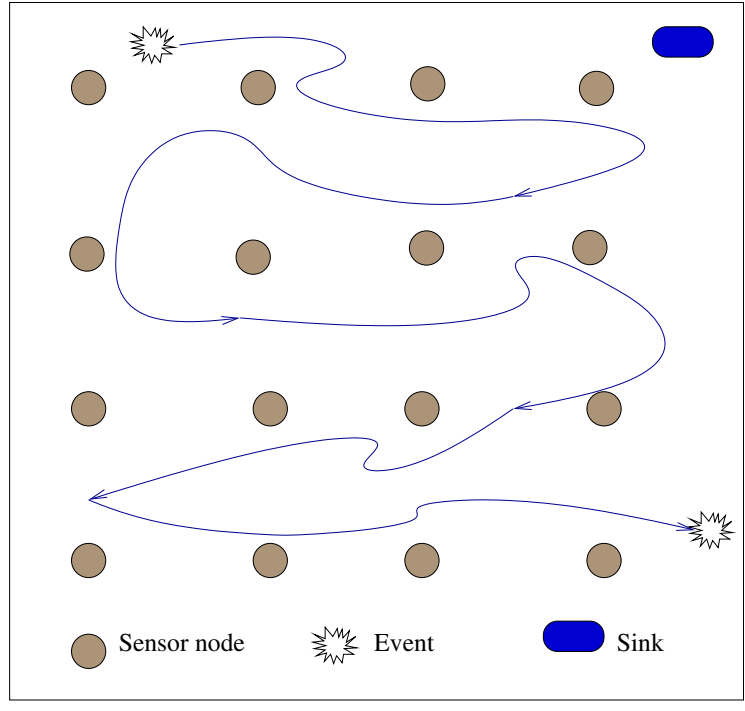

Figure 2. One pattern of event movement path.

to send data to the destination node, it first initiates route discovery process. In this process, source node broadcasts Route Request (RREQ) packet to its neighbors. Neighbor nodes which receive RREQ forward the packet to its neighbor nodes. Neighbor nodes which receive RREQ forward the packet to its neighbors, and so on. This process continues until RREQ reach to the destination or the node who know the path to destination. When the intermediate nodes receive RREQ, they record in their tables the address of neighbors, thereby establishing a reverse path. When the node which knows the path to destination or destination node itself receive RREQ, it send back Route Reply (RREP) packet to source node. This RREP packet is transmitted by using reverse path. When the source node receives RREP packet, it can know the path to destination node and it stores the discovered path information in its route table. That is the end of route discovery process. Then, AODV performs route maintenance process. In route maintenance process, each node periodically transmits a Hello message to detect link breakage.

\subsection{Propagation Radio Model}

In order to simulate the detection of a natural event, we used the libraries from Naval Research Laboratory (NRL) [10]. In this framework, a phenomenon is modeled as a wireless mobile node. The phenomenon node broadcasts packets with a tunable synchrony or pulse rate, which represents the period of occurrence of a generic event ${ }^{2}$. These li-

\footnotetext{
${ }^{2}$ As a consequence, this model is for discrete events. By setting a suitable value for the pulse rate, it is possible in turn to simulate the continuous
}

braries provide the sensor node with an alarm variable. The alarm variable is a timer variable. It turns off the sensor if no event is sensed within an alarm interval. In addition to the sensing capabilities, every sensor can establish a multihop communication towards the Monitoring Node (MN) by means of a particular routing protocol. This case is the opposite of the polling scheme.

Although not optimal for multi-hops WSNs, we assume that the MAC protocol is the IEEE 802.11 standard. This serves to us as a baseline of comparison for other contention resolution protocols. The receiver of every sensor node is supposed to receive correctly data bits if the received power exceeds the receiver threshold, $\gamma$. This threshold depends on the hardware ${ }^{3}$. As reference, we select parameters values according to the features of a commercial device (MICA2 OEM). In particular, for this device, we found that for a carrier frequency of $f=916 \mathrm{MHz}$ and a data rate of $34 \mathrm{KBaud}$, we have a threshold (or receiver sensitivity) $\left.\gamma\right|_{d B}=-118 \mathrm{dBm}[11]$. The calculation of the phenomenon range is not yet optimized and the phenomenon propagation is assumed to follow the propagation laws of the radio signals. Table 1 shows some typical values of Shadowing deviation. In Fig. 3 and Fig. 4 are shown the transimisson range of TwoRayGround and Shadowing models [12]. TwoRayGround model considers both the direct path and a ground reflection path. It is applied in the environments which are like plains and have no obstacles. However, the transmission range of Shadowing model is random. This model is applied in the environments which have obstacles and are hardly to transmit data directly. In particular, the emitted power of the phenomenon is calculated according to a TwoRayGround propagation model. The received power at distance $d$ is predicted by:

$$
P_{r}(d)=\frac{P_{t} G_{t} G_{r} h_{t}^{2} h_{r}^{2}}{d^{4} L}
$$

where $G_{t}$ and $G_{r}$ are the antenna gains of the transmitter and the receiver, $h_{t}$ and $h_{r}$ are the heights of the transmit and receive antennas respectively, and $L(L \geq 1)$ is the system loss.

The Shadowing model assumes that the received power at the sensor node is:

$$
\left.P_{r}(d)\right|_{\mathrm{dB}}=\underbrace{\left.P_{t}\right|_{\mathrm{dB}}-\beta_{0}-10 \alpha \log \left(\frac{d}{d_{0}}\right)}_{\text {deterministic part }}+\underbrace{S_{\mathrm{dB}}}_{\text {random part }}
$$

where $\beta_{0}$ is a constant. The term $S_{\mathrm{dB}}$ is a random variable, which accounts for random variations of the path loss. This

\footnotetext{
signal detection such as temperature or pressure.

${ }^{3}$ Other MAC factors affect the reception process, for example the Carrier Sensing Threshold (CST) and Capture Threshold (CP) of IEEE.802.11 used in ns-2.
} 


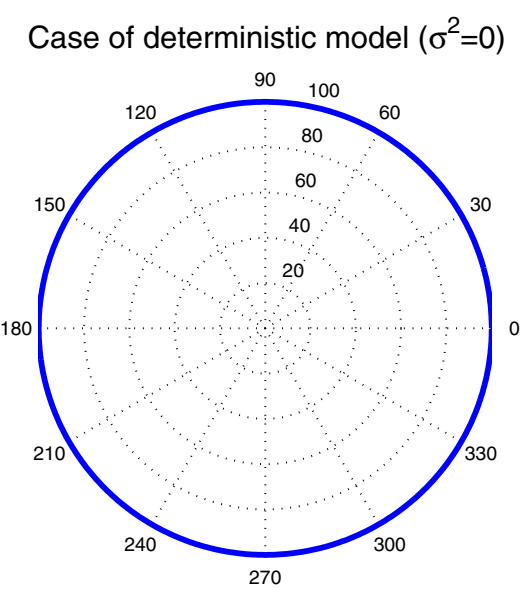

\section{Figure 3. Transimisson range of TwoRay- Ground model.}

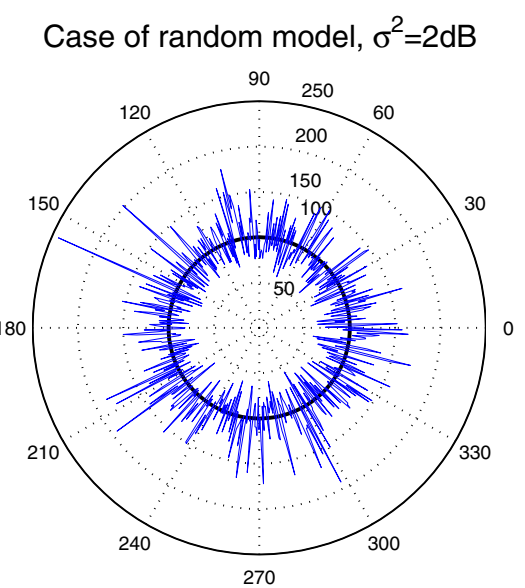

\section{Figure 4. Transimisson range of Shadowing} model.

variable is also known as log-normal shadowing, because it is supposed to be Gaussian distributed with zero mean and variance $\sigma_{\mathrm{dB}}^{2}$, that is $S_{\mathrm{dB}} \sim \mathcal{N}\left(0, \sigma_{\mathrm{dB}}^{2}\right)$. Given two nodes, if $P_{r}>\gamma$, where $\gamma$ is the hardware-dependent threshold, the link can be established. The case of $\sigma=0, \alpha=4$, $d>d_{0}$ is the TwoRaysGround model. In Shadowing model in addition to the direct ray from the transmitter towards the receiver node, a ground reflected signal is supposed to be present. Accordingly, the received power depends also on
Table 1. Some typical values of Shadowing deviation $S_{\mathrm{dB}}$.

\begin{tabular}{|c|c|}
\hline Environment & $S_{\mathrm{dB}}$ \\
\hline Outdoor & 4 to 12 \\
\hline Office, Hard partition & 7 \\
\hline Office, Soft partition & 9.6 \\
\hline Factory, Line-of-sight & 3 to 6 \\
\hline Factory, Obstructed & 6.8 \\
\hline
\end{tabular}

Table 2. Topology settings.

\begin{tabular}{|c|c|}
\hline \multicolumn{2}{|c|}{ Lattice } \\
\hline \hline Step & $d=\frac{L}{\sqrt{N}-1} \mathrm{~m}$ \\
\hline Service Area Size & $L^{2}=(800 \mathrm{x} 800) \mathrm{m}^{2}$ \\
\hline Number of Nodes & $N=12,64,100,256$ \\
\hline Transmission Range & $r_{0}=d$ \\
\hline
\end{tabular}

the antenna heights and the pathloss is:

$$
\beta=10 \log \left(\frac{(4 \pi d)^{4} L}{G_{t} G_{r} h_{t} h_{r} \lambda^{2}}\right)
$$

Energy Model The energy model concerns the dynamics of energy consumption of the sensor. A widely used model is as follows [13]. When the sensor transmits $k$ bits, the radio circuitry consumes an energy of $k P_{T x} T_{B}$, where $P_{T x}$ is the power required to transmit a bit which lasts $T_{B}$ seconds. By adding the radiated power $P_{t}(d)$, we have:

$$
E_{T x}(k, d)=k T_{B}\left(P_{T x}+P_{t}(d)\right) .
$$

Since packet reception consumes energy, by following the same reasoning, we have:

$$
\begin{aligned}
E(k, d) & =E_{T x}(k, d)+E_{R x}(k, d)=k P_{T x} T_{B}+k T_{B} P_{t}(d) \\
& +k P_{R x} T_{B}
\end{aligned}
$$

where $P_{R x}$ is the power required to correctly receive (demodulate and decode) one bit. In Tables 2 and 3, we summarise the values of parameters used in our WSNs. Let us note that the power values concern the power required to transmit and receive one bit, respectively. They do not refer to the radiated power at all. This is also the energy model implemented in the widely used ns- 2 simulator. It is take a long time to simulate a simulation system with large number of nodes. So, we investgated the performance of WSNs with the number of nodes shown in Table 2. 
Table 3. Radio model and system parameters.

\begin{tabular}{|c|c|}
\hline \multicolumn{2}{|c|}{ Radio model parameters } \\
\hline \hline Path Loss Coefficient & $\alpha=2.7$ \\
\hline Variance & $\sigma_{\mathrm{dB}}^{2}=16 \mathrm{~dB}$ \\
\hline Carrier Frequency & $916 \mathrm{MHz}$ \\
\hline Antenna & omni \\
\hline Threshold (Sensitivity) & $\gamma=-118 \mathrm{~dB}$ \\
\hline \multicolumn{2}{|c|}{ Other parameters } \\
\hline \hline Reporting Frequency & $T_{r}=[0.1,1000] \mathrm{pps}$ \\
\hline Interface Queue Size & 50 packets \\
\hline UDP Packet Size & 100 bytes \\
\hline Detection Interval $\tau$ & $30 \mathrm{~s}$ \\
\hline \multicolumn{2}{|c|}{ packet per seconds }
\end{tabular}

Interference In general, in every wireless network the electromagnetic interference of neighboring nodes is always present. The interference power decreases the Signalto-Noise-Ratio (SNR) at the intended receiver, which will perceives a lower bit and/or packet error probability. Given a particular node, the interference power depends on how many transmitters are transmitting at the same time of the transmission of the given node. In a WSNs, since the number of concurrent transmissions is low because of the low duty-cycle of sensors, we can neglect the interference. In other words, if we define duty-cycle as the fraction between the total time of all transmissions of sensor data and the total operational time of the net, we get always a value less than 0.5 . In fact, the load of each sensor is $\ll 1$ because sensors transmit data only when an event is detected [13]. However, it is intuitive that in a more realistic scenario, where many phenomena trigger many events, the traffic load can be higher, and then the interference will worsen the performance with respect to that we study here. Consequently, we can fairly say that the results we get here should be considered as an upper bound on the system performance with respect to more realistic scenarios.

\section{Event Detection and Transport}

Here, we use the data-centric model similar to [14], where the end-to-end reliability is transformed into a bounded signal distortion concept. In this model, after sensing an event, every sensor node sends sensed data towards the MN. The transport used is a UDP-like transport, i.e. there is not any guarantee on the delivery of the data. While this approach reduces the complexity of the transport protocol and well fit the energy and computational constraints of sensor nodes, the event-reliability can be guaranteed to some extent because of the spatial redundancy. The sensor

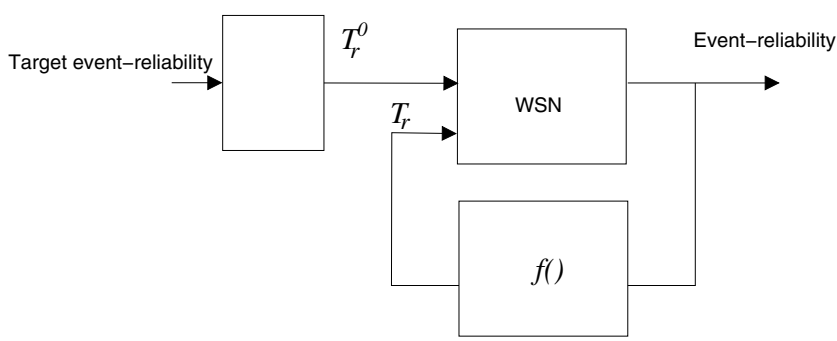

Figure 5. Representation of the transport based on the event-reliability.

node transmits data packets reporting the details of the detected event at a certain transmission rate ${ }^{4}$. The setting of this parameter, $T_{r}$, depends on several factors, as the quantization step of sensors, the type of phenomenon, and the desired level of distortion perceived at the MN. In [14], the authors used this $T_{r}$ as a control parameter of the overall system. For example, if we refer to event-reliability as the minimum number of packets required at $\mathrm{MN}$ in order to reliably detect the event, then whenever the $\mathrm{MN}$ receives a number of packets less than the event-reliability, it can instruct sensor nodes to use a higher $T_{r}$. This instruction is piggy-backed in dedicated packets from the MN. This system can be considered as a control system, as shown in Fig. 5, with the target event-reliability as input variable and the actual event-reliability as output parameter. The target event-reliability is transformed into an initial $T_{r}^{0}$. The control loop has the output event-reliability as input, and on the basis of a particular non-linear function $f(\cdot), T_{r}$ is accordingly changed. We do not implement the entire control system, but only a simplified version of it. For instance, we vary $T_{r}$ and observe the behavior of the system in terms of the mean number of received packets. In other words, we open the control loop and analyze the forward chain only.

\section{Goodput, Routing Efficiency and Consumed Energy}

In this section, we present the simulation results of our proposed WSNs. We simulated the network by means of ns2 simulator, with the support of NRL libraries ${ }^{5}$. The Goodput is defined at the sink, and it is the received packet rate divided by the sent packets rate. Thus:

$$
G(\tau)=\frac{N_{r}(\tau)}{N_{s}(\tau)}
$$

\footnotetext{
${ }^{4}$ Note that in the case of discrete event, this scheme is a simple packet repetition scheme.

${ }^{5}$ Since the number of scheduler events within a simulated WSNs can be very high, we applied a patch against the scheduler module of ns-2 in order to speed up the simulation time [15].
} 
where $N_{r}(\tau)$ is the number of received packet at the sink, and the $N_{s}(\tau)$ is the number of packets sent by sensor nodes which detected the phenomenon. Note that the eventreliability is defined as $G_{R}=\frac{N_{r}(\tau)}{R(\tau)}$, where $R$ is the required number of packets or data in a time interval of $\tau$ seconds.

We defined the RE parameter as the ratio of sent packets from sensing node with sent packet by routing protocol. Thus:

$$
R E(\tau)=\frac{N_{\text {sent }}(\tau)}{N_{\text {routing }}(\tau)}
$$

where $N_{\text {routing }}(\tau)$ is the number of sent packets by routing protocol, and $N_{\text {sent }}(\tau)$ is the number of sent packets by sensor nodes which detect the phenomenon.

As long as the WSNs is being used, a certain amount of energy will be consumed. The energy consumption rate directly affects the life-time of the network, i.e. the time after which the WSNs is unusable. The energy depletion is a function of the reporting rate as well as the densigy of the sensor network. Recall that the density of the network in the event-driven scenario correlates with the number of nodes that report their data. Accordingly, we define the consumed energy by the network in the detection interval $\tau$ as:

\section{Consumed Energy $\triangleq$}

$$
\frac{\text { Initial T. Energy - Final T. Energy }}{\tau} .
$$

However, in order to compare the performances of the scaled networks, it is better to define the mean energy depletion rate per node as:

$$
\bar{\Delta}(\tau) \triangleq \frac{E_{I}-\bar{e}(\tau)}{\tau}=\frac{N E_{I}-\sum_{i=1}^{N} e_{i}(\tau)}{N \tau}
$$

where $e_{i}(t)$ is the node energy at time $t$ and the means are computed over the number of nodes. The number of nodes $N$ is set as power of integers in order to analyse the behaviour of the scaled versions of the network. The initial position of the phenomenon node is varied along the simulation runs.

\section{Simulation Results}

For AODV routing protocol, the sample averages of Eqs. (5), (6) and (8) are computed over 20 simulation runs, and they are plotted in Fig. $6 \sim$ Fig. 11, with respect to the particular radio model used.

In Fig. $6 \sim$ Fig. 9 we can clearly distinguish three operating zones. For low values of $T_{r}$, the network is uncongested. At a particular value of $T_{r}(\sim 1 \mathrm{pps})$, the Goodput arise abruptly, because the network has reached the maximum capacity. For $T_{r}>1 \mathrm{pps}$, contention and congestion periods augment, increasing $T_{r}$ does not ameliorate the Goodput and $N_{r}(\tau)$ is roughly constant. In case of both TwoRayGround the Goodput decreases with N, as shown in Fig. 6 and Shadowing in Fig. 7, respectively. However, we found that the Goodput of TwoRayGround is better than Shadowing. The consumed energy of Shadowing is about half of the TwoRayGound model as shown in Fig. 8 and Fig. 9. The explanation of this effect is not simple, because it is intermingled with the dynamics of MAC and routing protocol. However, intuitively we can say that in the case of Shadowing the on-demand routing protocols are affected by the presence of shadowing-induced unidirectional links. It is worth noting that AODV and other protocol cannot use unidirectional links. On the other hand, exploiting such links is possible but the performance gains are quite low. Thus, the routing protocol spends most of the time in the searching of a bi-directional path. Thus, given a fixed detection interval, $N_{r}$ can be much lower than its value in the case of ideal radio model, i.e. the Two-Rays-Ground model, where the discovered paths do not change over time ${ }^{6}$. This fact may not affect the performance of the WSNs, because it depends on the requirements of the application. For high values of $N$, the augmented interference level and the path instability seem to be predominant [16].

In Fig. 10 and Fig. 11 is shown the average value of RE using TwoRayGround and Shadowing radio models in case of mobile event. The RE is an increasing function of $T_{r}$, because as $T_{r}$ increases, the number of sent packet by sensing node is higher than the number of packets used by routing protocol. From the results, we found the RE of TwoRayGround is better than Shadowing.

\section{Conclusions}

In this paper, we presented the implementation of a simulation system for WSNs using ns-2. We used AODV protocol and carried out the simulations for mobile event considering two cases: TwoRayGround and Shadowing.

- The Goodput of TwoRayGround is better than Shadowing, and the Goodput of TwoRayGround does not change too much when the number of nodes is increased. However, the Goodput of Shadowing is decreased much more with the increase of number of nodes.

- The depletion of Shadowing is better than TwoRayGround in case of mobile event.

\footnotetext{
${ }^{6}$ This is true if we do not count the reliability of nodes, i.e. the probability of failure of sensor nodes.
} 


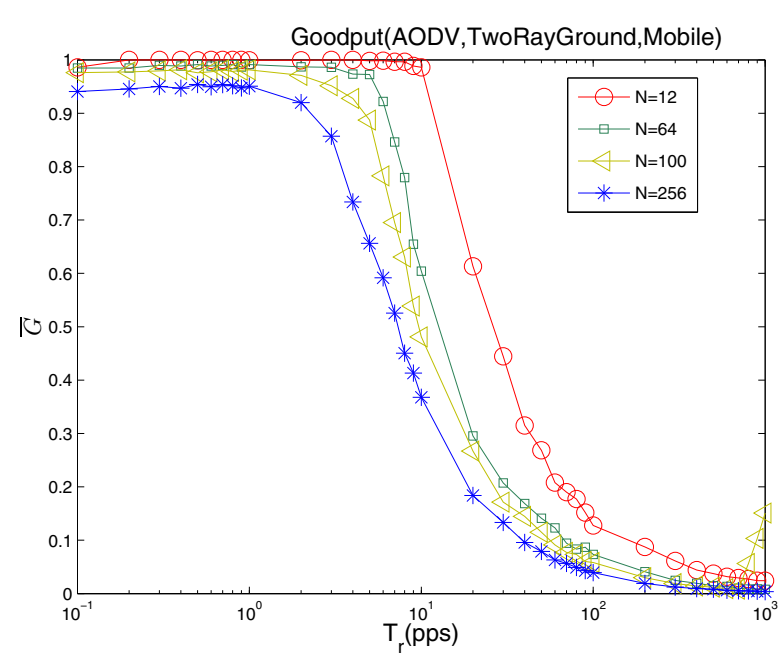

Figure 6. Goodput for mobile event with TwoRayGround.

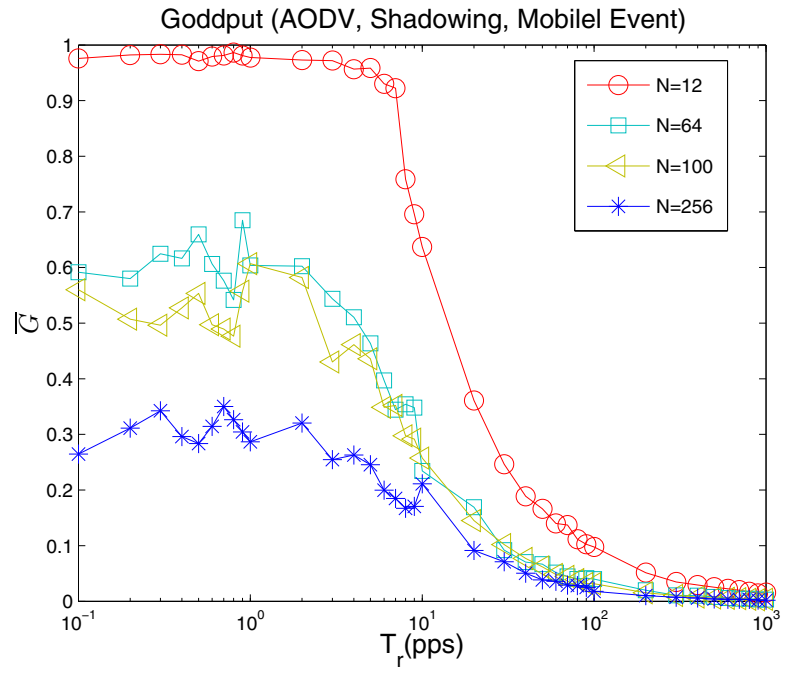

Figure 7. Goodput for mobile event with Shadowing.

- The RE of Shadowing is better than TwoRayGround when the number of nodes is small (12 nodes). However, when the number of nodes increases the RE of TwoRayGround is better than Shadowing.

In the future, we would like to carry out more extensive simulations for mobile sensor nodes and mobile sink. We also would like to carry out simulations for sensor and actor networks.

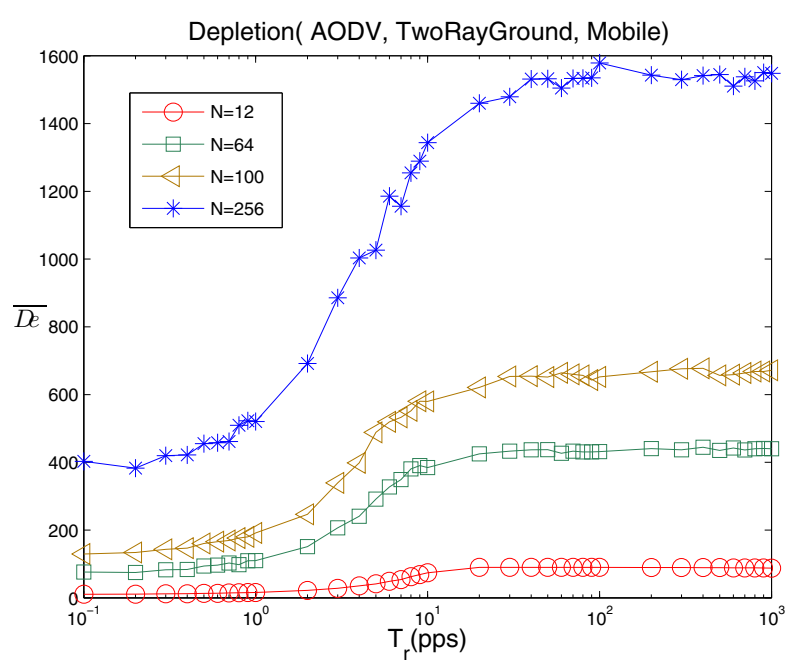

Figure 8. Depletion for mobile event with TwoRayGround.

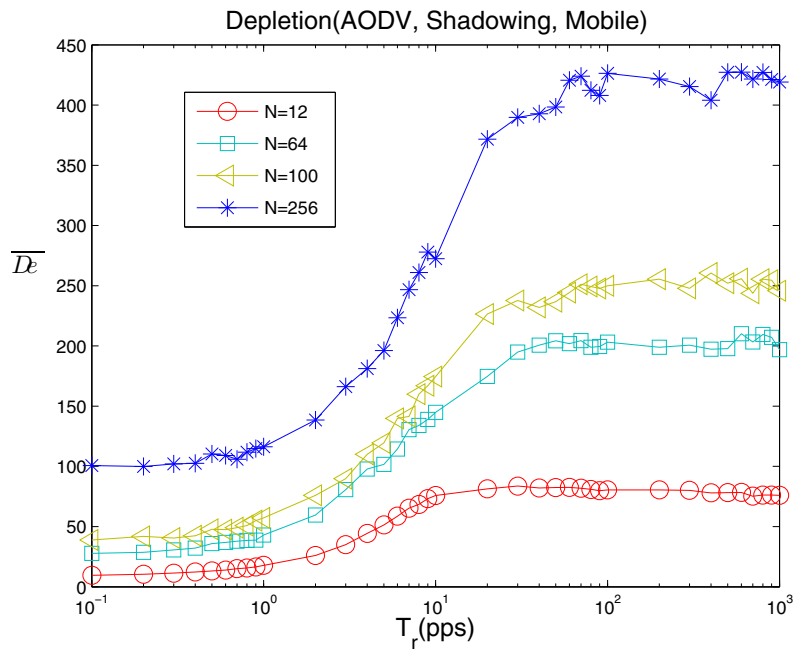

Figure 9. Depletion for mobile event with Shadowing.

\section{Acknowledgment}

The authors would like to thank International Communications Foundation (ICF) of Japan and Japanese Society for the Promotion of Science (JSPS) for supporting this work.

\section{References}

[1] I. F. Akyildiz and I. H. Kasimoglu, "Wireless sensor and actor networks: research challenges", Ad Hoc 


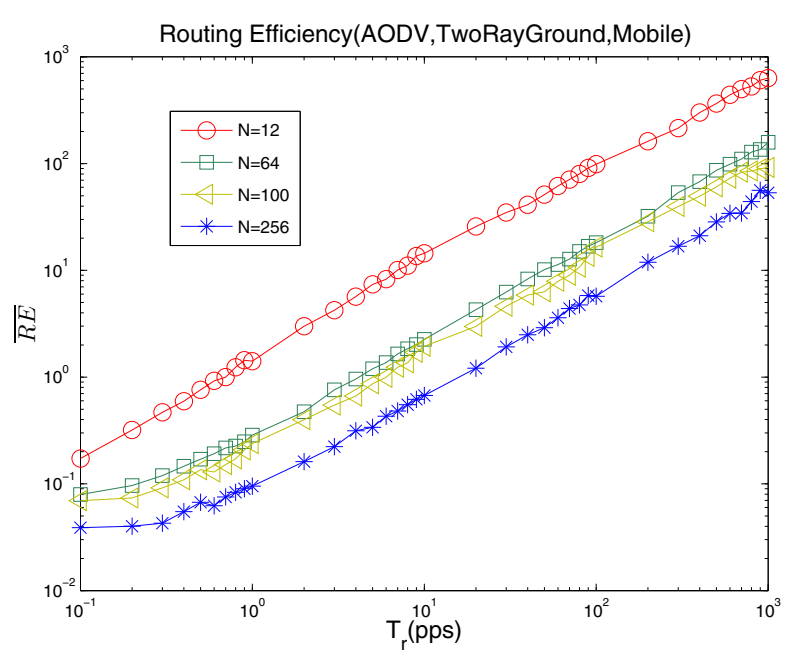

Figure 10. RE for mobile event with TwoRayGround.

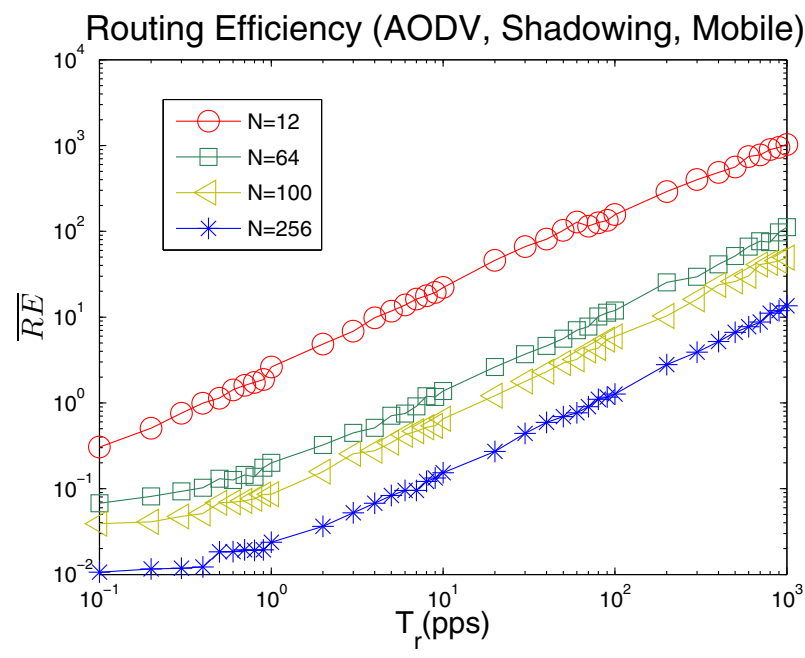

Figure 11. RE for mobile event with Shadowing.

Networks Journal (Elsevier), Vol. 2, No. 4, pp. 351367, October 2004.

[2] O. Younis and S. Fahmy, "HEED: A hybrid, energyefficient, distributed clustering approach for ad-hoc sensor networks", IEEE Transactions on Mobile Computing, Vol. 3, No. 4, pp. 366-379, October 2004.

[3] S. Giordano and C. Rosenberg, "Topics in Ad Hoc and Sensor Networks", IEEE Communication Magazine, Vol. 44, No. 4, pp. 97-97, 2006.
[4] J. N. Al-Karaki and A. E. Kamal, "Routing Techniques in Wireless Sensor Networks: A Survey", IEEE Wireless Communication, Vol. 11, No. 6, pp. 6-28, December 2004.

[5] T. Yang, G. De Marco, M. Ikeda, and L. Barolli, "A case study of event detection in lattice wireless sensor network with shadowing-induced radio irregularities", Proc. of The Fourth International Conference on Advances in Mobile Computing and Multimedia (MoMM), pages 241-250, December 2006.

[6] G. De Marco, T. Yang, L. Barolli, "Impact of Radio Irregularities on Topology Tradeoffs of WSNs", Proc. of NBiS-2006/DEXA-2006 (Krakow, Poland), pp. 5054, September 2006.

[7] G. W.-Allen, K. Lorincz, O. Marcillo, J. Johnson, and M. Ruiz an J. Lees, "Deploying a wireless sensor network on an active volcano", IEEE Internet Computing, Vol. 10, No. 2, pp. 18-25, March 2006.

[8] C. Cooper. A note on the connectivity of 2-regular digraphs. Random Structures Algorithms, Vol. 4, No. 4, pp. 469-472, 1993.

[9] C. Perkins, editor, Ad Hoc Networks, AddisonWesley, 2001.

[10] Network simulator, http://www.isi.edu/nsnam/ns/.

[11] Crossbow technology, inc. http://www.xbow.com/.

[12] T.S. Rappaport, Wireless Communications, Prentice Hall PTR, 2001.

[13] W. Ye, J. Heidemann and D. Estrin, "Medium access control with coordinated adaptive sleeping for wireless sensor networks", IEEE/ACM Transaction Networking, Vol.12, No. 3, pp. 493-506, June 2004.

[14] Özgür B. Akan and I. F. Akyildiz, "Event-tosink reliable transport in wireless sensor networks", IEEE/ACM Transactions on Networking, Vol. 13, No. 5, pp. 1003-1016, October 2005.

[15] D. X. Wei, "Speeding up ns-2 scheduler", http://netlab.caltech.edu/ weixl/technical/ns2patch, September 2005. California Institute of Technology.

[16] G. Zhou, T. He, S. Krishnamurthy and J. A. Stankovic, "Models and solutions for radio irregularity in wireless sensor networks", ACM Transaction on Sensors Network, Vol. 2, No. 2, pp. 221-262, May 2006. 